\title{
Projections of preventable risks for cardiovascular disease in Canada to 2021: a microsimulation modelling approach
}

\author{
Douglas G. Manuel MD MSc, Meltem Tuna PhD, Deirdre Hennessy PhD, Carol Bennett MSc, \\ Anya Okhmatovskaia PhD, Philippe Finès PhD, Peter Tanuseputro MD MSc, Jack V. Tu MD PhD, \\ William Flanagan MSc; for the Simulation Technology for Applied Research Team and the Canadian \\ Cardiovascular Outcome Research Team
}

See related commentary in CMAJ by Egger and colleagues at www.cmaj.ca/lookup/doi/10.1503/cmaj.140138

\section{Abstract}

Background: Reductions in preventable risks associated with cardiovascular disease have contributed to a steady decrease in its incidence over the past 50 years in most developed countries. However, it is unclear whether this trend will continue. Our objective was to examine future risk by projecting trends in preventable risk factors in Canada to 2021.

Methods: We created a population-based microsimulation model using national data on births, deaths and migration; socioeconomic data; cardiovascular disease risk factors; and algorithms for changes in these risk factors (based on sociodemographic characteristics and previous cardiovascular disease risk). An initial population of 22.5 million people, representing the Canadian adult population in 2001, had 13 characteristics including the risk factors used in clinical risk prediction. There were 6.1 million potential exposure profiles for each person each year. Outcome measures included annual prevalence of risk factors (smoking, obesity, diabetes, hypertension and lipid levels) and of co-occurring risks.

Results: From 2003 to 2009, the projected risks of cardiovascular disease based on the microsimulation model closely approximated those based on national surveys. Except for obesity and diabetes, all risk factors were projected to decrease through to 2021. The largest projected decreases were for the prevalence of smoking (from $25.7 \%$ in 2001 to $17.7 \%$ in 2021) and uncontrolled hypertension (from 16.1\% to 10.8\%). Between 2015 and 2017, obesity was projected to surpass smoking as the most prevalent risk factor.

Interpretation: Risks of cardiovascular disease are projected to decrease modestly in Canada, leading to a likely continuing decline in its incidence.

\footnotetext{
t $t$ is uncertain whether the incidence of cardiovascular disease (CVD) in Canada will increase, decrease or stabilize in the foreseeable future. This lack of insight is surprising, given the steady and remarkable $70 \%$ decline in incidence over the past 50 years of a disease that accounts for the death of more than 70000 Canadians annually. ${ }^{1}$

In Canada and other developed countries, the uncertainty surrounding the incidence of CVD is largely a consequence of opposing trends associated with preventable risks. Some prominent CVD risk factors have declined steadily. Notably, the prevalence of tobacco smoking in Canada has decreased from $50 \%$ in 1995 to about $17 \%$ in $2011 .^{2}$ Similarly, the prevalence of uncontrolled hypertension has declined more than $70 \%$ since 1992. ${ }^{3}$ However, obesity rates have been steadily rising and may overshadow the advances made in decreasing other preventable risks, resulting in a reversal of gains in life expectancy. ${ }^{4}$
}

We sought to project the prevalence of risk factors for CVD in Canada to 2021. Because CVD is influenced by multiple risk factors, we projected prevalence trends for both individual and multiple co-occurring risks. We also modelled social and demographic characteristics that are associated with CVD risks, including future changes in the structure of the Canadian population.

Competing interests: None declared.

This article has been peer reviewed.

Correspondence to: Carol Bennett, cbennett@ohri.ca

CMAJ Open 2014.DOI:10.9778/cmajo.20120015 


\section{Methods}

\section{Population Health Model framework}

We generated projections of the prevalence of CVD risk factors using a Canadian microsimulation model: the Population Health Model (POHEM) for CVD. In brief, Statistics Canada's POHEM is an empirically grounded, longitudinal microsimulation model of multiple diseases and risk factors representing the life-cycle dynamics of the Canadian population. ${ }^{5}$ Its basic unit of analysis is the individual person. Through dynamic simulation, POHEM creates a population and ages it, one person at a time, until death. In the CVD model, the life trajectory of each simulated person unfolds to create a life course reflecting his or her CVD-related risks. Each person's CVD risk is estimated annually with the use of individual, predictive risk equations that consider his or her characteristics in the previous year(s), including age, sex, other sociodemographic characteristics and health behaviour. The predictive equations are derived from a range of Canadian data sources (described in Appendix 1, available at www.cmaj open.ca/content/2/2/E94/suppl/DC1). POHEM-CVD is part of a larger POHEM framework that can project disease incidence, prevalence, life expectancy, health-adjusted life expectancy and quality of life for multiple chronic diseases. The main covariables modelled are summarized in Appendix 2.

\section{Study population}

The study population represented all Canadian residents, aged 20 years and older, living in a community setting from 2001 to 2021.

\section{Model characteristics}

The Simulation Technology for Advanced Research and the Canadian Cardiovascular Outcome Research teams created model specifications, which defined the purpose of the model, its overall structure and data sources. The main characteristics of the CVD model included: (a) population-based (reflecting the Canadian population, including important factors such as age, sex, socioeconomic position and province); (b) open population (allowing the population to change over time, reflecting births, deaths, immigration and emigration); (c) relevant risk factors (including individual CVD risks that have an important population burden or are used for estimation of future risk of CVD events and including categories or levels of exposure that are commonly used in risk stratification); (d) multiple risk factors (enabling projection of population exposure to multiple co-occurring risk factors); (e) predictive accuracy (allowing generation of accurate [well-calibrated] projections for the total Canadian population as well as specific subgroups based on sex, age and socioeconomic position); (f) useful for population health planning (allowing estimation of the health benefit of prevention scenarios, including their influence on future CVD events).

\section{Model development}

The microsimulation model was developed in 4 steps: creation of the initial population, annual updates, model validation (and calibration if needed) and projection (Figure 1). Canadian population-based data sources were used for model initialization, yearly updates (including the generation of risk algorithms) and validation (Figure 2 and Appendix 1).

Initial population: We created the initial population for the POHEM-CVD model using the 2000/01 Canadian Community Health Survey. Each respondent, aged 20 years or more $(n=105908)$, was replicated with use of his or her survey weight to generate a cohort so that the total simulated population equaled the approximately 22.5 million communitydwelling Canadians in 2001 (see Appendix 1 for details about the Canadian Community Health Survey).

Data on CVD risks that were not available in the Canadian Community Health Survey (i.e., measured systolic and diastolic blood pressure and lipid levels [total cholesterol and high-density lipoprotein cholesterol]) were imputed from the 1990 Canadian Heart Health Survey with the use of validated "hot-deck" methods based on age group, sex, body mass index (BMI) category and diabetes status (see e'Table 3.2 in Appendix 3). ${ }^{6-9}$ Because levels of medical treatment were low in 1990, Canadian Heart Health Survey data allowed us to project the lifetime course of treatment of relatively untreated blood pressure and lipid levels. Treated levels of blood pressure and lipids were then incorporated separately during model calibration to reflect the substantial uptake in treatment since 1990 (see section on validation and calibration below).

Each person in the POHEM population had 13 characteristics: 5 demographic characteristics (age, sex, region of residence, ethnicity and immigrant status); 2 socioeconomic characteristics (income and education); 4 proximal risks (diabetes, heart disease, blood pressure and lipid levels); and 2 health behaviours (change in BMI and smoking). The CVD risk factors were classified by level of exposure typically used in clinical prediction of CVD risk. Five major factors (blood pressure, lipid levels, BMI, diabetes and smoking) had 2-5 exposure levels, for a total of 600 combinations of CVD risk exposure (Appendices 2 and 4). Along with other characteristics, the result was a total of 6.14 million potential states or exposure profiles.

Annual updates and risk transitions: Five steps were required to update the model each year from 2001 to 2021 (Figure 2). The population structure was updated by aging each person 1 year and changing the total population size based on births, deaths and immigration/emigration data. Education and family income were projected. Finally, each person's CVD risks were updated by applying predictive algorithms for CVD risk change (described below). See Appendix 5 for details .

Separate predictive algorithms were developed for each CVD risk, except for blood pressure and lipid level, for which the same algorithm was used. With each annual model update, the algorithms moved some people from 1 category of health risk exposure to another: for example, transition from nonsmoker to current smoker, transition from current smoker to former smoker or transition to new-onset diabetes. Each algorithm had a different set of predictors. All algorithms included age and sex, as well as up to 7 additional predictors (Appendix 
2). All algorithms were generated with the use of Canadian population data. Specifically, we modelled change in BMI using the longitudinal National Population Health Survey from 1996/97 to 2004/05. We modelled change in smoking status using the Canadian Health Survey (1979), the National Population Health Survey (1994) and the Canadian Community Health Survey (2008). We estimated diabetes risk using the Diabetes Population Risk Tool,${ }^{10}$ which was developed with data from the 1996/97 National Population Health Survey and validated with data from the 2001 Canadian Community Health Survey linked to a population-based diabetes registry. We modelled changes in blood pressure and lipid levels using the cross-sectional Canadian Heart Health Survey (19861992). Whether a period, actuarial or life-table approach is used, the transition of blood pressure and cholesterol values from 1 age group to the next is assumed to follow a life-course perspective and therefore can be used to estimate change in these risk factors over time. In brief, the transport flow method, with the SAS NETFLOW procedure, was used to estimate the transition probabilities of blood pressure and cholesterol levels by diabetes status and BMI. See Appendices 1 and 3 for a more detailed description of the data sources and methods for updating all CVD risks.

Validation and calibration: The projected prevalences of the CVD risk factors obtained from POHEM-CVD were validated against observed estimates from the Canadian Community Health Survey and the Canadian Health Measures Survey. Four cycles of the Canadian Community Health Survey conducted between 2003 and 2009 were used for validation of smoking, diabetes and obesity. Although similar to the Canadian Community Health Survey (1.1) used to initialize POHEM, these later surveys provide external validation because they represent a cross-section of the Canadian population at different times. External validation is a preferred method for establishing model credibility. ${ }^{11}$ The Canadian Health Measures Survey, which collected biophysical measures, was carried out twice (2007-2009 and 2009-2011), allowing for validation and calibration of blood pressure and lipid levels. Predicted births, deaths and immigration numbers were compared with Statistics Canada's observed counts compiled from provincial vital statistics (see Appendix 5 for details). Observed and projected estimates were compared for multiple risk factors for Canadians overall.

The model was considered well calibrated if the difference between values predicted by the model and population estimates from the Canadian Community Health Survey or the Canadian Health Measures Survey was less than 5\%. Because of small sample sizes in these surveys, for people 75 years of age or older, we accepted a difference of less than $10 \%$. For any particular CVD risk, our a priori calibration approach was

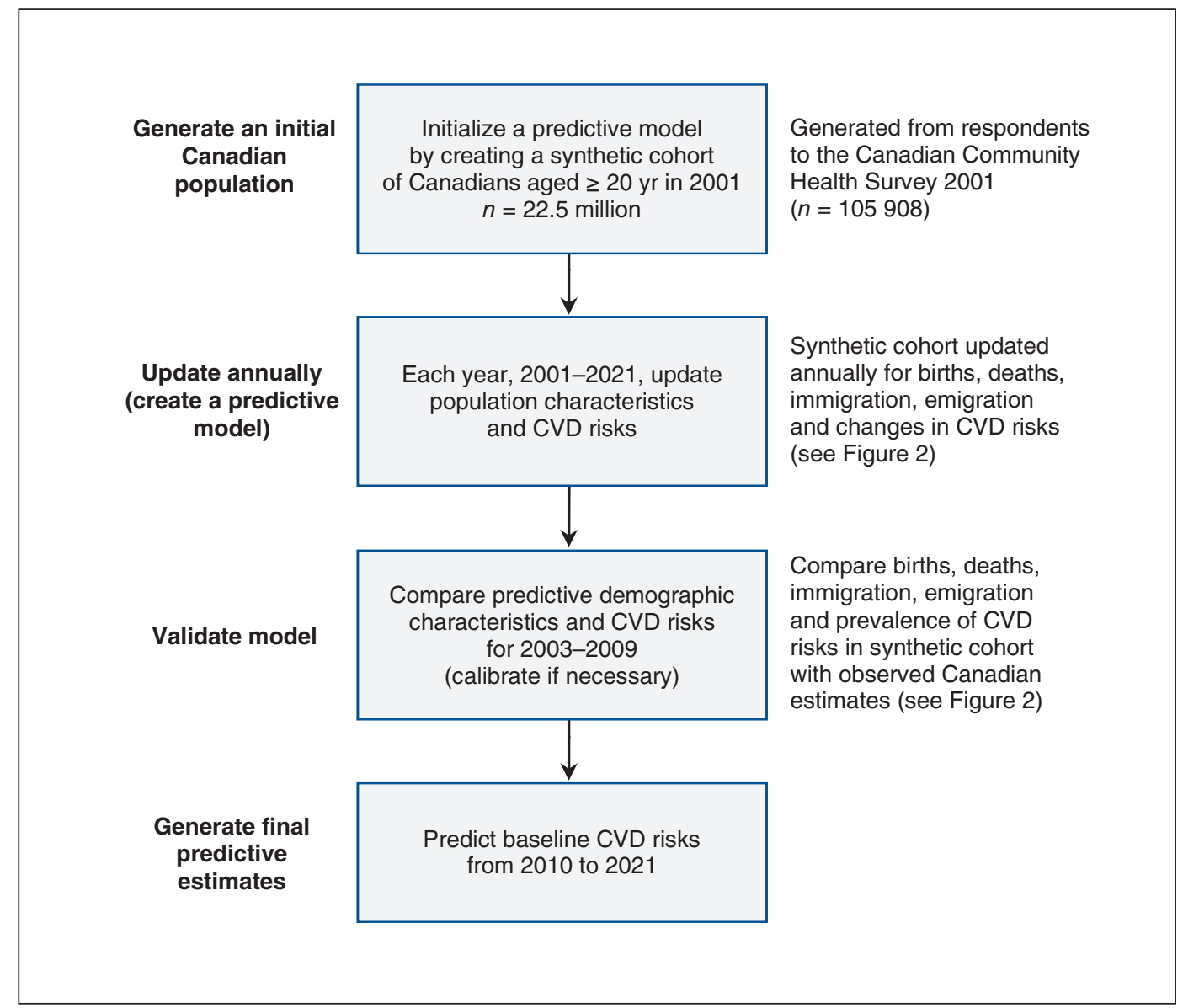

Figure 1: Process for projecting baseline risks of cardiovascular disease (CVD) in Canada from 2010 to 2021. 
to adjust model estimates if more than half the strata-specific estimates exceeded the calibration cutoff point (i.e., $5 \%$ or $10 \%$ difference). In this case, the model estimates were adjusted to match the survey estimates. Calibration can be carried out as part of estimation in the microsimulation model or afterward (outside the model). In this study, we took the approach of adjusting estimates outside the model. This calibration approach was proposed and approved during model specification with the Simulation Technology for Advanced Research and the Canadian Cardiovascular Outcome Research teams.

Projection: The model projected CVD risks for each person in the population from 2003 to 2021. The projections were then aggregated by year for predefined subgroups, based on demographic characteristics. Trends observed in the various Canadian population data used to generate the algorithms were applied to future projections. The projections thus reflect the "baseline" trends in health and societal factors that affect CVD risk.

\section{Microsimulation modelling and statistical analysis}

The POHEM-CVD risk model was generated with the use of Modgen (www.statcan.gc.ca/microsimulation/modgen /modgen-eng.htm), a microsimulation programming language developed and supported by Statistics Canada. Statistical analysis of data from the National Population Health Survey,

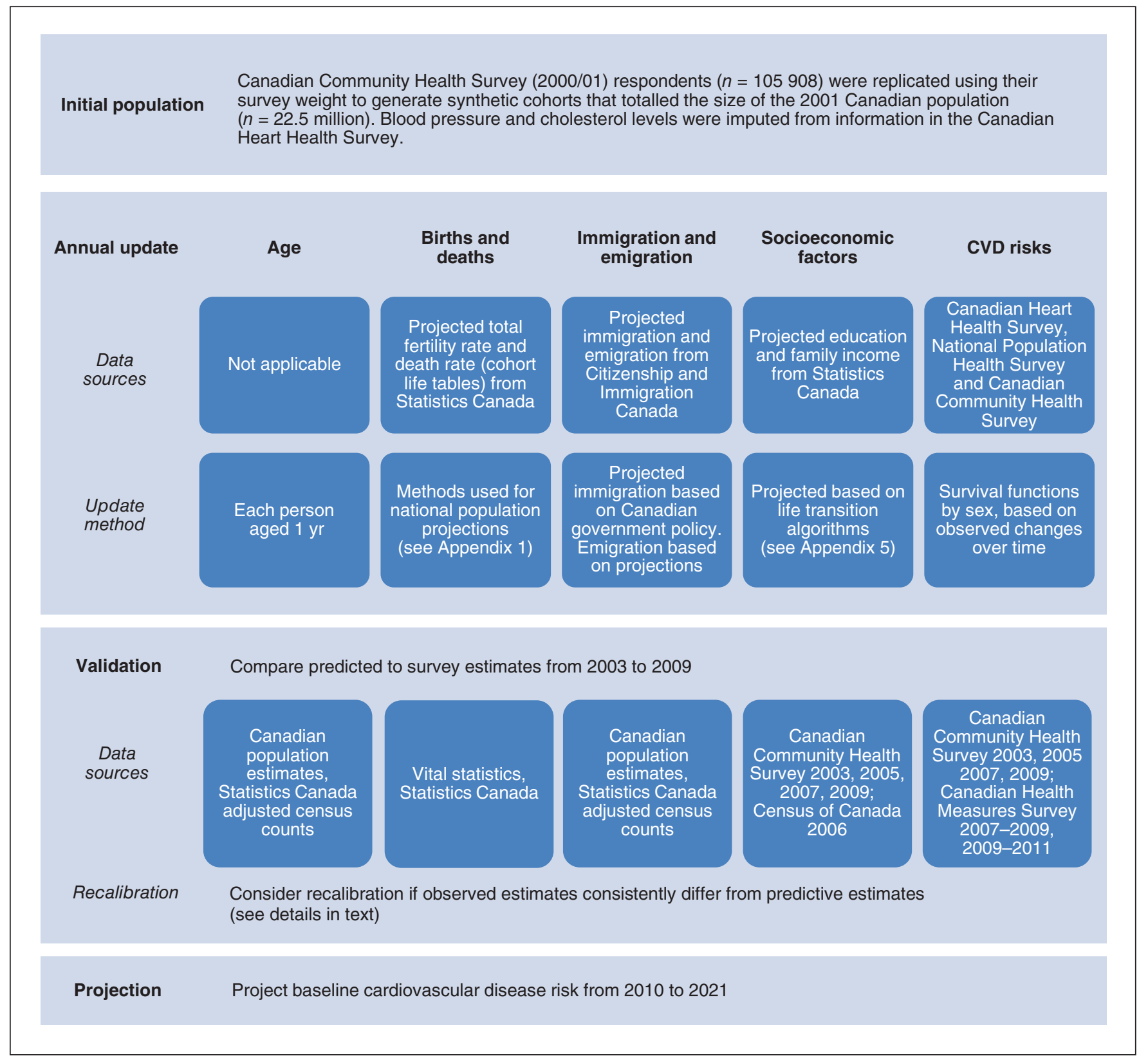

Figure 2: Summary of data sources and methods used to generate and validate projections of cardiovascular disease (CVD) risks in Canada, 2001-2021. 
the Canadian Community Health Survey, the Canadian Health Measures Survey and the Canadian Heart Health Survey was carried out with the use of SAS.

\section{Results}

Table 1 shows the prevalence of 5 CVD risk factors in the simulated Canadian population in 2001: current smoking, obesity (BMI $\geq 30$ ), hypertension (systolic blood pressure $\geq 140 \mathrm{~mm} \mathrm{Hg}$ ), high cholesterol levels (total cholesterol $\geq 6.22 \mathrm{mmol} / \mathrm{L}$ ) and diabetes. Current smoking was the most common factor (28.4\% of men and $23.0 \%$ of women) and approximately twice as prevalent as obesity $(15.9 \%$ of men and $14.4 \%$ of women), the next most common risk factor. Among men, 14.2\% had high total cholesterol levels, $16.0 \%$ were hypertensive, and $5.0 \%$ had diabetes. The risk profile for women was similar $(14.6 \%$ had high total cholesterol levels, $16.1 \%$ were hypertensive, and $4.4 \%$ had diabetes).

The model was well-calibrated for both the initial 2001 population and for 2003-2009 projections (Figures 3 and 4), except for hypertension and high total cholesterol level. Calibration of these projections was required, because they were more than 5\% higher than observed in the 2007-2009 and 2009-2011 Canadian Health Measures Surveys. Specifically, POHEM estimates of age- and sex-specific hypertension and high cholesterol levels were adjusted by the corresponding subgroup differences between the model and survey estimates.

Figure 3 shows projected estimates of risk prevalence to 2021 , along with survey data and $95 \%$ confidence intervals from 2001 to 2009, for smoking, obesity, diabetes, high total cholesterol levels and hypertension. Obesity was projected to increase and, between 2015 and 2017, overtake smoking as the most prevalent CVD risk factor.

Figure 4 shows a projected, slight decrease in exposure to any 1 of 3 risk factors: obesity, smoking and diabetes. The number of people with all 3 of these risk factors was low throughout the period ( $0.3 \%$ in 2001 declining to $0.2 \%$ in 2021).

\section{Interpretation}

This study projected to 2021 an overall modest decline in the prevalence of most CVD risk factors in Canada, with the exception of diabetes and obesity, which are shown to increase. Smoking, hypertension and high total cholesterol level had large projected declines. The projected reduction in smoking is concordant with widely implemented smoking prevention strategies across Canada; for example, by 2007, most provinces had prohibited smoking in public indoor spaces. ${ }^{12}$ In addition, the control of hypertension has increased 4-fold in Canada (from $13 \%$ in 1992 to $65 \%$ in 2009 ) ? $^{3}$ and statin medications for dyslipidemia are the leading treatment prescribed in government-funded drug plans. ${ }^{13}$

Projections of the prevalence of CVD risk factors should be interpreted with care. We generated them using data available in 2001 (the baseline), and we derived risk-transition algorithms from other historic data. The actual future prevalence of risk factors beyond the validation period (i.e., after 2009) will undoubtedly vary from our projections. The prevalence of risk factors could be lower than our projections if healthy physical and social conditions improve or if efforts to prevent hypertension and dyslipidemia are strengthened. Conversely, Canada's physical and social environment may become more conducive to an increase in obesity. The primary use of population projection models, such as POHEM-CVD, is analogous to the use of predictive models at the clinical level - namely, to inform decision-making by presenting plausible future outcomes, including before and after implementation of possible interventions and treatments. ${ }^{14}$ In this way, the POHEM-CVD model can be used to examine the potential consequences of preventive strategies, such as increasing coverage of blood pressure treatment or implementing a new community-wide smoking policy, given the known or postulated effectiveness of such strategies.

The next logical step in our research will be to project the incidence of diseases related to these risk factors. In addition to using similar microsimulation techniques, we could project future CVD incidence by combining the risk factor estimates from the POHEM-CVD model with the risk hazard estimates used in historic CVD projection models, such as that of Wijeysundera and colleagues. ${ }^{15}$ Alternatively, we could

\begin{tabular}{|c|c|c|}
\hline \multicolumn{3}{|c|}{$\begin{array}{l}\text { Table 1: Characteristics and prevalence of risk factors } \\
\text { for cardiovascular disease in the simulated } 2001 \text { Canadian } \\
\text { population }\end{array}$} \\
\hline Characteristic & $\begin{array}{c}\text { Men, } \% \\
n=11000000^{*}\end{array}$ & $\begin{array}{c}\text { Women, \% } \\
n=11500000^{*}\end{array}$ \\
\hline \multicolumn{3}{|l|}{ Age, yr } \\
\hline $20-44$ & 52.9 & 50.2 \\
\hline $45-64$ & 32.7 & 32.0 \\
\hline $65-74$ & 9.1 & 10.0 \\
\hline$\geq 75$ & 5.3 & 7.8 \\
\hline Current smoker & 28.4 & 23.0 \\
\hline \multicolumn{3}{|l|}{ Body mass index } \\
\hline$<18.5$ (underweight) & 1.2 & 4.5 \\
\hline 18.5-24.9 (normal) & 42.6 & 54.0 \\
\hline 25-29.9 (overweight) & 40.2 & 27.1 \\
\hline$\geq 30$ (obese) & 15.9 & 14.4 \\
\hline \multicolumn{3}{|l|}{ Systolic blood pressure, $\mathrm{mm} \mathrm{Hg}$} \\
\hline < 120 (optimal) & 46.9 & 61.8 \\
\hline 120-129 (normal) & 23.6 & 12.9 \\
\hline 130-139 (high-normal) & 13.5 & 9.2 \\
\hline$\geq 140$ (hypertensive) & 16.0 & 16.1 \\
\hline \multicolumn{3}{|l|}{ Total cholesterol, mmol/L } \\
\hline$<4.15$ (low) & 17.1 & 17.4 \\
\hline 4.15-5.17 (low-medium) & 35.5 & 37.3 \\
\hline 5.18-6.21 (medium) & 33.2 & 30.8 \\
\hline$\geq 6.22$ (high) & 14.2 & 14.6 \\
\hline Diabetes diagnosis & 5.0 & 4.4 \\
\hline
\end{tabular}


incorporate a CVD incidence algorithm, such as the Framingham Risk Tool. ${ }^{16}$ In either case, we expect CVD incidence - adjusted for Canada's larger, aging population - to decrease through to 2021. POHEM-CVD projects a decrease in smoking, uncontrolled hypertension and dyslipi- demia, each of which confers a 2- to 3-fold increased risk of CVD. Diabetes constitutes a similar hazard and is projected to increase, influencing an upward trend in CVD incidence. However, the projected increase in the prevalence of diabetes is considerably smaller than the projected decrease in other

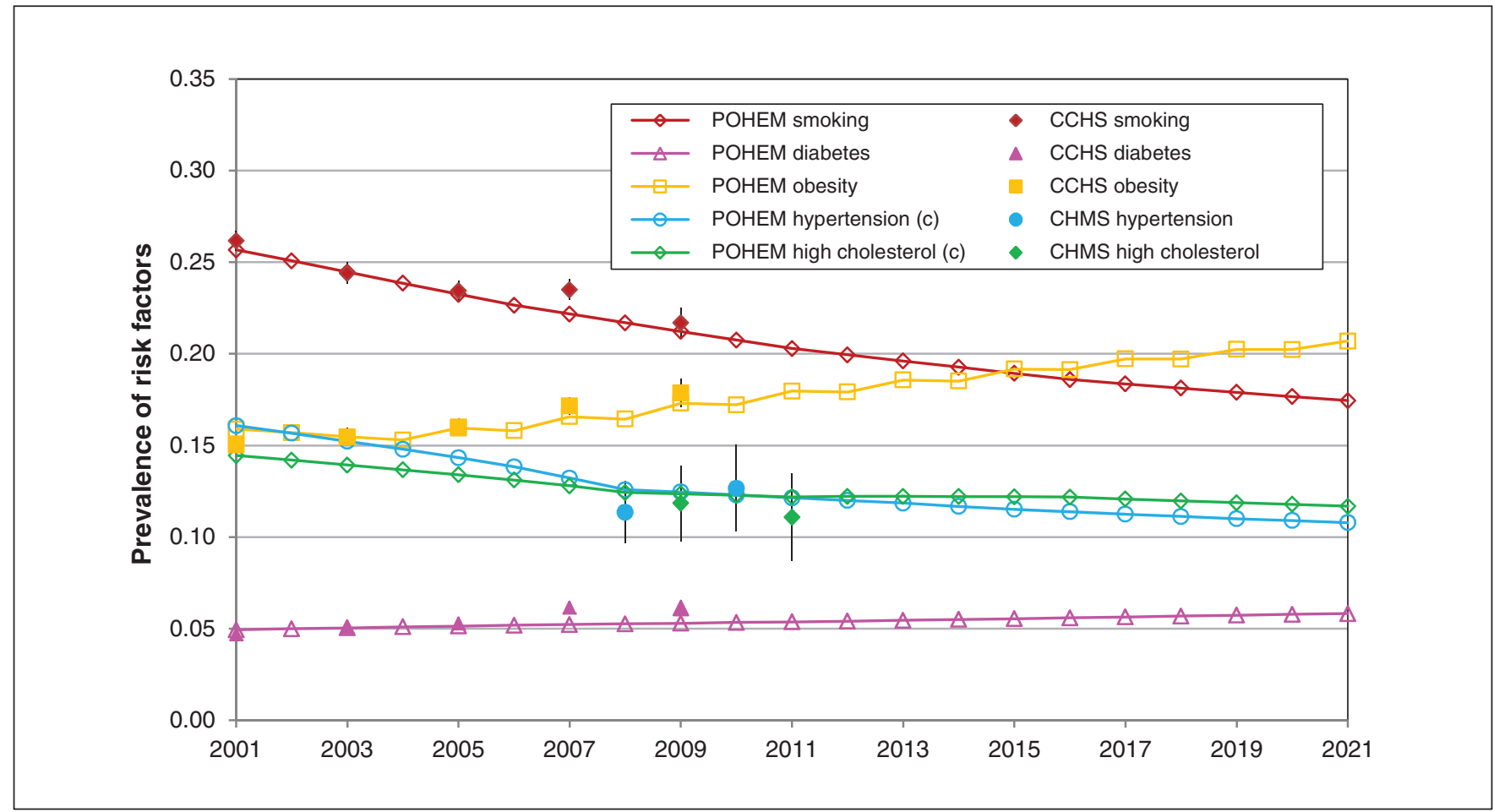

Figure 3: Prevalence of cardiovascular disease risk factors in the Canadian population: projected estimates from the microsimulation model (POHEM) and observed estimates from Canadian Community Health Survey (CCHS) and Canadian Health Measures Survey (CHMS) data; $95 \%$ confidence intervals (error bars) are shown for the survey data. Obesity = body mass index $\geq 30$, hypertension $=$ systolic blood pressure $\geq 140 \mathrm{~mm} \mathrm{Hg}$, high cholesterol = total cholesterol $\geq 6.22 \mathrm{mmol} / \mathrm{L}$. *POHEM projections for hypertension and high cholesterol level are calibrated.

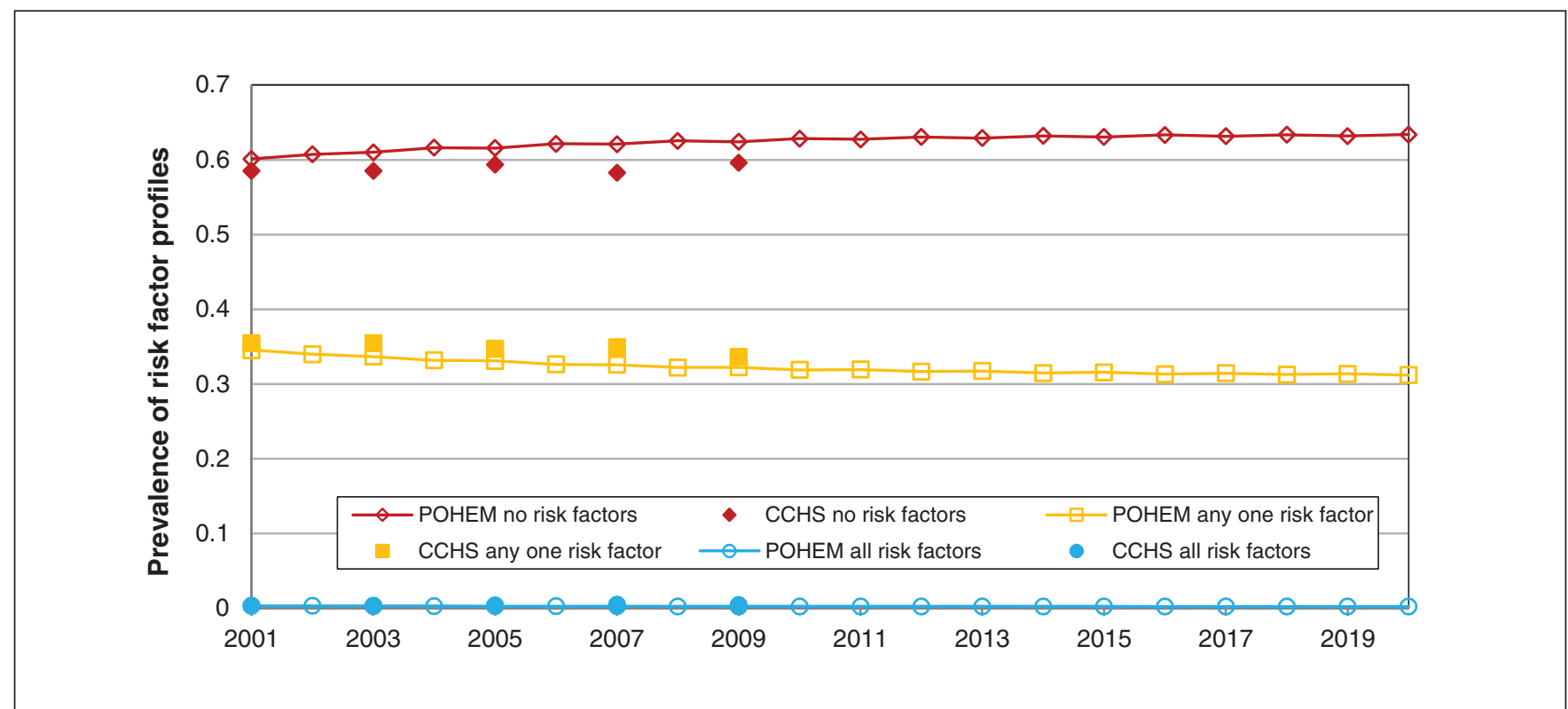

Figure 4: Prevalence of no risk factors; any of smoking, obesity and diabetes; and all risk factors in the Canadian population projected using the microsimulation Population Health Model (POHEM) and estimated from the Canadian Community Health Survey (CCHS). 
major CVD risks. The prevalence of obesity will also increase notably; however, obesity confers a comparatively smaller risk of CVD than other factors. ${ }^{17}$

\section{Strengths and limitations}

Our study has 2 main strengths. First, POHEM-CVD uses predictive algorithms for CVD risk changes similar to those used for clinical algorithms, except that they are generated with the use of population-based data. A microsimulation approach allows for projections of risk factors based on multiple predictors and open populations that change with births, deaths and migration. It also allows projections of multiple coevolving risks across varied strata (e.g., age, sex and socioeconomic position). The ability of microsimulation models to incorporate complexity is particularly important for conditions such as CVD that have multiple, interacting risk factors that lead to a protracted course of illness; this represents an important advance over other modelling approaches. ${ }^{18,19}$

Second, we validated our projections against external data. This approach has become common in clinical risk prediction but is not widely used in population settings. Our projected CVD risk estimates compared closely with external estimates from the Canadian Community Health Survey (2003-2009) and the Canadian Health Measures Survey (2011), except for blood pressure and total cholesterol level, where uncalibrated projections exceeded survey estimates. The need to calibrate for blood pressure and cholesterol was expected because of the known strong trend in better treatment in Canada and the United States. ${ }^{3,20}$

Accurate or well-calibrated projection of BMI, smoking prevalence and diabetes status during the validation period (2003-2009) was possible because these CVD risk factors appear to be largely predictable over the short- or medium-term based on sociodemographic characteristics and related health behaviours. ${ }^{21-23}$ For example, a person's current BMI is closely related to previous measurements and will generally reflect the population life course of this measure, that is, an increase up to middle age, followed by a slow decrease in older age. ${ }^{21}$ The POHEMCVD model captures these life-course patterns.

Our study has several limitations, including the inherent challenge of projecting CVD risks that are influenced by constantly changing social environments and health care systems. Although we included many CVD risks, notable omissions in POHEM-CVD included physical activity, sedentary activity, diet, stress and biophysical markers (e.g., apolipoprotein B and C-reactive protein). However, compared with other studies, we included a larger range of empirically derived risk and exposure levels..$^{24,25}$ Of particular note, we considered the major CVD risks that are important for predicting CVD incidence. It has been debated whether including additional risk factors would improve the prediction or characterization of CVD incidence; however, generally, calibration has resulted in no or very minor incremental improvement with the addition of risks beyond those included in POHEM-CVD. ${ }^{26}$

Another limitation of our study is the lack of comprehensive longitudinal data for physical measures, such as cholesterol levels and blood pressure over time. The Canadian Heart Health Survey, carried out in the 1990s, provided the only data on physical measures available for the Canadian population until the advent of the Canadian Health Measures Survey. In our study, the projections for cholesterol levels and blood pressure were based on the cross-sectional Canadian Heart Health Survey data. As data from more cycles of the Canadian Health Measures Survey become available, they will be used for validation, calibration and to estimate treatment effects for selected risk factors from the 1990s to the present. In addition, future studies will use the most recent Canadian Community Health Survey data, for 2010 and beyond, for validation.

\section{Conclusion}

Our results suggest that, except for obesity and diabetes, CVD risk factors will continue to decline in Canada. The prevalence of people with multiple CVD risk factors will remain low. Projecting CVD risks using a microsimulation approach can inform decision-making with the goal of continued reductions in CVD risk and incidence. The next step in our research will be to use the POHEM-CVD model to project CVD incidence. Given the trends in the prevalence of CVD risk factors shown in our study, particularly the combined effect of notable decreases in the prevalence of smoking and other risks, CVD incidence will likely continue to decline. However, the projected increases in obesity and diabetes will likely attenuate, but not reverse, decreases in CVD incidence.

\section{References}

1. Tracking heart disease and stroke in Canada. Ottawa (ON): Public Health Agency of Canada; 2009.

2. Reid JL, Hammond D, Burkhalter R, et al. Tobacco use in Canada: patterns and trends, 2013 edition. Waterloo (ON): Propel Centre for Population Health Impact, University of Waterloo; 2013. Available: www.tobaccoreport.ca/2013 /TobaccoUseinCanada_2013.pdf (accessed 2014 Apr. 24).

3. McAlister FA, Wilkins K, Joffres M, et al. Changes in the rates of awareness, treatment and control of hypertension in Canada over the past two decades. CMA7 2011;183:1007-13.

4. Olshansky SJ, Passaro DJ, Hershow RC, et al. A potential decline in life expectancy in the United States in the 21st century. N Engl 7 Med 2005; 352:1138-45.

5. Wolfson MC. POHEM - a framework for understanding and modelling the health of human populations. World Health Stat Q 1994;47:157-76.

6. Durrant GB. Imputation methods for handling item-nonresponse in the social sciences: a methodological review. Southampton (UK): ESRC National Centre for Research Methods and Southampton Statistical Sciences Research Institute, University of Southampton; 2005.

7. Durrant GB, Skinner C. Using data augmentation to correct for non-ignorable non-response when surrogate data are available: an application to the distribution of hourly pay. 7 R Stat Soc Ser A Stat Soc 2006;169:605-23.

8. Durrant GB, Skinner C. Using missing data methods to correct for measurement error in a distribution function. Surv Methodol 2006;32:25-36.

9. Hennessy D, Bennett C, Tuna M, et al. An assessment of methods to impute risk exposure into model actor's risk profile for microsimulation. In: 2011 International Methodology Symposium: Strategies for standardization of methods and tools - how to get there; 2011 Nov 1-4; Ottawa (ON). Ottawa: Statistics Canada; 2011. Internal document; available on request.

10. Rosella LC, Manuel DG, Burchill C, et al. PHIAT-DM team. A populationbased risk algorithm for the development of diabetes: development and validation of the Diabetes Population Risk Tool (DPoRT). 7 Epidemiol Community Health 2011;65:613-20.

11. Kopec JA, Finès P, Manuel DG, et al. Validation of population-based disease simulation models: a review of concepts and methods. BMC Public Health 2010;10:710.

12. Public smoking bans by province and territory. Ottawa (ON): Health Canada; 2007.

13. Drug use among seniors on public drug programs in Canada, 2002 to 2008. Ottawa (ON): Canadian Institute for Health Information; 2010.

14. Barlow J, Bayer S. Raising the profile of simulation and modelling in health services planning and implementation. F Health Serv Res Policy 2011;16:129-30.

15. Wijeysundera HC, Machado M, Farahati F, et al. Association of temporal trends in risk factors and treatment uptake with coronary heart disease mortality, 1994-2005. . AMA 2010;303:1841-7. 
16. Sheridan S, Pignone M, Mulrow C. Framingham-based tools to calculate the global risk of coronary heart disease: a systematic review of tools for clinicians. f Gen Intern Med 2003;18:1039-52.

17. Wilson PW, D'Agostino RB, Sullivan L, et al. Overweight and obesity as determinants of cardiovascular risk: the Framingham experience. Arch Intern Med 2002;162:1867-72.

18. Zucchelli E, Jones AM, Rice N. The evaluation of health policies through microsimulation methods. Heslington, York (UK): University of York; 2010. Health Economics and Data Group working paper 10/03. Available: www.york.ac.uk /media/economics/documents/herc/wp/10_03.pdf (accessed 2014 Mar. 22).

19. Spielauer M. What is dynamic social science microsimulation? Ottawa (ON): Statistics Canada. Available: www.statcan.gc.ca/microsimulation/pdf/chap1-eng.pdf (accessed 2012 Nov. 1).

20. Joffres M, Shields M, Tremblay MS. Dyslipidemia prevalence, treatment, control, and awareness in the Canadian Health Measures Survey. Can 7 Public Health 2013;104:e252-7.

21. Orpana HM, Tremblay MS, Finès P. Trends in weight change among Canadian adults. Health Rep 2007;18:9-16.

22. Shields M. Smoking bans: influence on smoking prevalence. Health Rep 2007; 18:9-24.

23. Ross NA, Gilmour H, Dasgupta K. 14-year diabetes incidence: the role of socio-economic status. Health Rep 2010;21:19-28.

24. Ainsworth JD, Carruthers E, Couch P, et al. IMPACT: a generic tool for modelling and simulating public health policy. Methods Inf Med 2011;50:454-63.

25. Capewell S, Critchley J, Unal B. IMPACT, a validated, comprehensive coronary beart disease model. Liverpool (UK): University of Liverpool; 2007.

26. Diamond GA. What price perfection? Calibration and discrimination of clinical prediction models. 7 Clin Epidemiol 1992;45:85-9.

Affiliations: Statistics Canada (Manuel, Tuna, Hennessy, Finès, Flanagan); Ottawa Hospital Research Institute (Manuel, Tuna, Hennessy, Bennett, Tanuseputro); Department of Family Medicine (Manuel), University of Ottawa, Ottawa, Ont.; Institute for Clinical Evaluative Sciences (Tuna,
Bennett, Tanuseputro), Ottawa and Toronto, Ont.; Department of Epidemiology, Biostatistics and Occupational Health (Okhmatovskaia), McGill University, Montréal, Que.; Bruyère Research Institute (Tanuseputro), Bruyère Centre of Learning, Research and Innovation in Long-Term Care, Ottawa, Ont.; Department of Health Policy (Tu), Management, and Evaluation, University of Toronto, Toronto, Ont.

Contributors: Douglas Manuel, Meltem Tuna, Carol Bennett and William Flanagan contributed substantially to the conception and design of the study. Meltem Tuna, Deirdre Hennessy, Carol Bennett and Anya Okhmatovskaia contributed to acquisition of the data. All of the authors were involved in analysis and interpretation of the data. Douglas Manuel, Meltem Tuna, Deirdre Hennessy and Carol Bennett drafted or revised the article. All of the authors approved the final version submitted for publication and agreed to act as guarantors of the work.

Funding: The study was supported by funding from the Canadian Institutes of Health Research through the Canadian Cardiovascular Outcome Research Network and the New Emerging Team in Simulation Technology for Applied Research (FRN: ETG-92246). The Population Health Model (POHEM) was developed by, and receives support from, Statistics Canada and partnering agencies. Douglas Manuel holds a Chair in Applied Population Health from the Canadian Institutes of Health Research and the Public Health Agency of Canada. Jack Tu is supported by a Canada Research Chair in Health Services Research and a Career Investigator Award from the Heart and Stroke Foundation of Ontario.

Supplemental information: For reviewer comments and the original submission of this manuscript, please see www.cmajopen.ca/content $/ 2 / 2$ /E94/suppl/DC1 